\title{
Estudio térmico en una vivienda situada en la ciudad de Guayaquil
}

DOI: https://doi.org/10.33262/ap.v3i4.1.124

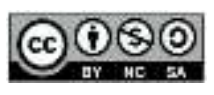

\section{Thermal study in a villa located in the city of Guayaquil}

\author{
Holger Arturo Intriago Mairongo. ${ }^{1}$, Alex Andrés Gonzales Vega. ${ }^{2}$, Egner Manuel \\ Rosero Obando. ${ }^{3} \&$ Felipe Noé Plaza Hernández. ${ }^{4}$
}

\begin{abstract}
The construction sector is one of the sectors that has the greatest impact on the country's economy the present work seeks to determine in a quantitative exploratory way the most influential parameters in the thermal behavior of a house located in a very hot humid climate, for this in the work a study of heat flow in the walls, floor and ceiling of a housing to try to reduce the energy consumption of air conditioning and advertise them as ecoefficient in the city of Guayaquil - Ecuador, using common construction materials of the houses, according to the latest INEC census, these materials are: concrete block $15 \mathrm{~cm}$ thick for walls, $15 \mathrm{~cm}$ thick lightened concrete on the ceiling and $20 \mathrm{~cm}$ heavy concrete on the floor; furthermore, $20 \%$ of the wall surfaces are glazed with $6 \mathrm{~mm}$ thick single glass windows. For this, the heat gains from walls and ceilings will be evaluated at noon, three hours before and after noon, for a typical day due to the effect of sensible heat. Through the application of numerical methods and with the assistance of the octave free
\end{abstract}

\footnotetext{
1 Universidad Luis Vargas Torres, Facultad Mecánica, Esmeraldas, Ecuador holger.intriago@utelvt.edu.ec, https://orcid.org/0000-0003-3694-1219

2 Universidad Luis Vargas Torres, Facultad Mecánica, Esmeraldas, Ecuador alex.gonzalez.vega@utelvt.edu.ec, https://orcid.org/0000-0002-3785-0442

${ }^{3}$ Universidad Técnica Luis Vargas Torres, Facultad Mecánica, Esmeraldas, egner.rosero@utelvt.edu.ec, https://orcid.org/0000-0003-4994-4128

${ }^{4}$ Universidad Luis Vargas Torres, Facultad Mecánica, Esmeraldas, Ecuador, felipe.plaza@utelvt.edu.ec, https://orcid.org/0000-0002-0433-4724
} 
software, it is determined that the color gain depends on the orientation of the house with respect to the sun and the largest wall area

Keywords: thermal, housing, eco-efficient, convection, conduction.

\section{Resumen}

El sector de la construcción es uno de los sectores que mayor impacto tiene en la economía del país; el presente trabajo busca determinar de forma exploratoria cuantitativa los parámetros más influyentes en el comportamiento térmico de una vivienda ubicada en un clima húmedo muy caluroso, para ello en el trabajo se realizó un estudio de flujo de calor en las paredes, piso y techo de una vivienda para tratar de disminuir el consumo energético del aire acondicionado y anunciarlas como eco-eficiente en la ciudad de Guayaquil - Ecuador, empleando materiales de construcción comunes de las viviendas, de acuerdo con el último censo del INEC, estos materiales son: bloque de concreto de 15 $\mathrm{cm}$ de espesor para paredes, concreto alivianado de $15 \mathrm{~cm}$ de espesor en el techo y concreto pesado de $20 \mathrm{~cm}$ en el piso; además, el $20 \%$ de las superficies de las paredes son acristaladas con ventanas de vidrio simple de $6 \mathrm{~mm}$ de espesor. Para ello se evaluarán las ganancias de calor por paredes y techos al medio día, tres horas antes y después del mediodía, para un día típico por efecto del calor sensible. Mediante la aplicación de métodos numéricos y con asistencia del software libre octave, se determina que la ganancia de color depende de la orientación de la vivienda con respecto al sol y del área más grande de pared.

Palabras clave: térmico, vivienda, eco-eficiente, convección, conducción.

\section{Introducción}

El sector de la construcción es uno de los sectores que mayor impacto tiene en la economía del país; así mismo la vivienda tiene gran importancia ya que se transforma en las bases en donde se desarrolla el núcleo familiar, creando sentimientos de pertenencia y de confort. Es por ello que todo núcleo familiar merece tener una vivienda en donde pueda ver crecer su hogar, sintiendo que vive en un lugar digno y de calidad. La realidad del país, prevalece en el déficit de vivienda, en especial en los sectores económicos medios bajos y bajos. Es un reto, tanto para el gobierno, como para el sector inmobiliario, disminuir este déficit implementando viviendas que puedan ser de precios económicos, pero que al mismo instante sean capaces de brindar seguridad y calidad a sus ocupantes según lo afirma (Olmedo, 2017).

A partir del estudio de la termodinámica, se ha aprendido que la energía se puede transferir por interacciones de un sistema con su entorno. Estas interacciones se denominan trabajo y calor. Sin embargo, la termodinámica se ocupa de los estados finales del proceso durante el cual una interacción ocurre y no proporciona información sobre la naturaleza del entorno el tiempo índice en lo que ocurre. 
Los estudios que se realizan a nivel de eficiencia energética para el desafío del emprendedor en construcciones de viviendas eficientes requiere de cambios conceptuales de fondo en varios aspectos como: el diseño, uso de materiales y aplicación de sistemas constructivos innovadores, que satisfagan las necesidades de los usuarios en las edificaciones y que sean sustentables con el medio ambiente propone como "ecología arquitectónica" según el mismo autor, los edificadores de viviendas son responsables de un elevado consumo de energía, por lo cual se ve la necesidad latente de aplicar nuevos conceptos enmarcados dentro de los estudias relacionados con la trasferencia de calor (Filippín, 2010).

Existen diferentes factores que ayudan a determinar el confort como son: los factores internos y factores externos, en lo que respecta a los factores externos podemos decir que se refiere al grado de arropamiento, tipo y color de la vestimenta, factores ambientales como temperatura de aire, temperatura radiante, humedad del aire, velocidad de viento, niveles lumínicos, niveles acústicos, calidad del aire, olores, ruidos elementos visuales, etc. (Alvear, 2021), considerando la diversidad a la que se refiere se puede subdividir en dos tipos: los factores físicos o climáticos en lo que se encuentra la temperatura del aire, radiación, humedad en el movimiento del aire; y los factores individuales que abarcan la vestimenta, aclimatación, sexo, edad, forma del cuerpo, grasa subcutánea, alimentos bebidas, color de la piel y estado de salud.

En la actualidad en el mundo existe un déficit de viviendas de interés social en la ciudad de Guayaquil Ecuador los grandes asentamientos humanos generan la necesidad de construir casas que de alguna forma solucionen el hacinamiento que existen en las zonas urbanas. El presente estudio tiene como objetivo, determinar las ganancias de calor de una vivienda ubicada en la ciudad de Guayaquil, con el fin de plantear propuestas que nos permitan disminuir el consumo energético del aire acondicionado y presentar una solución viable como vivienda eco-eficiente. Para el análisis del comportamiento de esta vivienda se emplea los métodos numéricos mediante el uso de un software libre muy eficiente como lo es e Octave, el cual mediante la caracterización des variables que intervienen en el comportamiento térmico de la vivienda bajo las condiciones iniciales para luego realizar la modelación numérica; esto nos permitirá definir que materiales son los más apropiados para evitar que la vivienda gane demasiado calor en condiciones de temperaturas muy elevados que caracterizan a esta ciudad (A. Calle, 2021).

Pregunta de investigación: ¿Cuál es la ganancia de calor de una vivienda expuesta a las temperaturas en la ciudad de Guayaquil?

Objetivo, evaluar bajo el método numérico las ganancias de calor en una vivienda construida con materiales comunes, ubicada en la ciudad de Guayaquil en un clima húmedo caluroso durante el mediodía, tres horas antes y después del mediodía.

\section{Objetivos especificos:}

- Definir las condiciones iniciales de la vivienda para realizar el analizar las ganancias de calor. 
- Realizar el modelamiento numérico para determinar las ganancias de calor de una vivienda ubicada en la ciudad de Guayaquil.

- Calcular el valor de las resistencias total por convección y conducción.

- Definir los materiales con mejor características de conductividad térmica para disminuir la ganancia de calor

\section{Marco teórico}

Ecuador presenta escasez cualitativa y cuantitativa de conjuntos habitacionales para familias de bajos recursos son alarmantes, la limitación de servicios masicos afecta a un número importante de hogares de la región. El $21 \%$ no dispones de instalaciones hidrosanitarias y eléctricas; $12 \%$ de las viviendas están fabricadas de materiales inadecuados, otro porcentaje que fluctúa en $6 \%$ disponen de un suelo de tierra o viven en condiciones de aglomeración, estas características perjudican directamente la salud (C. Calle, 2021).

En la actualidad desde sus inicios la ciudad de Guayaquil se ha caracterizado por tener un clima tropical promedio durante todo el año, dando temperaturas que van desde $24{ }^{0} \mathrm{C}$. mínimo y $30{ }^{0} \mathrm{C}$ máximo, esta condicionante climática genera temperaturas inestables dentro de edificaciones unifamiliares, construidas con materiales tradicionales. En la ciudad de Guayaquil existen edificaciones unifamiliares construidas con materiales de construcción que no poseen propiedades térmicas, en la mayoría de los casos esto se debe a la falta de aplicaciones de materiales adecuados, para la construcción de una envolvente que genere confort térmico en las edificaciones multifamiliares (Medina, 2016).

\section{Propiedades térmicas}

Las propiedades térmicas se refieren a la mayor o menor capacidad de transmitir calor o acumularlo, y así dar lugar a la inercia térmica de una construcción según dichas capacidades se pueden definir como: densidad, calor especifico y conductividad térmica, que se refiere al material también está la transmitancia térmica, la capacidad calorífica y el retardo que se refieren a los elementos constructivos, como los cerramientos horizontales (techos), los cerramientos verticales transparentes (paños fijos, ventanas y puertas) y los cerramientos verticales opacos (muros y puertas) (Estevez, 2021).

El presente trabajo se describen los parámetros de entrada, considerados para el modelado de vivienda datos meteorológicos y datos monitoreados interiores necesarios para llevar a cabo el modelo matemático del cálculo de ganancia de calor, luego se describe la forma en que fueron variados los parámetros de entrada para obtener un modelo calibrado, una vez calculado se procedió a la evaluación del confort térmico.

Para la evaluación se estableció el modelo para calcular los límites de temperatura operativa en base as horas donde se determinó la mayor incidencia radiación solar en la vivienda (INCROPERA, 2011). 
Para continuar con la evaluación se determinaron los flujos de calor a través de la envolvente de las viviendas y conocer los elementos que aportan calor al interior de la vivienda

\section{Resistencia térmica}

En este punto observamos que, para el caso especial de transferencia de calor unidimensional sin generación de energía interna y con propiedades constantes, se sugiere un concepto muy importante por la ecuación 1 (INCROPERA, 2011). En particular, existe una analogía entre la difusión de calor y eléctrico cargar. Así como una resistencia eléctrica se asocia con la conducción de electricidad, una resistencia térmica puede estar asociada con la conducción de calor. Definiendo resistencias como la relación entre un potencial impulsor y la tasa de transferencia correspondiente, se sigue de Ecuación 3.4 que la resistencia térmica para la conducción en una pared plana es:

$$
q_{x}=-k A \frac{d T}{d x}=\frac{k A}{L}\left(T_{s, 1}-T_{s, 2}\right) \quad \text { Ecu. } 1 .
$$

Fuente: INCROPERA (2011)

Nota: Fundamentals of heat and mass transfer seventh edition "Frank P. Incropera / David P. Dewitt"

Para el desarrollo del cálculo numérico de la vivienda ubicada en la ciudad de Guayaquil, se utilizó software libre OCTAVE con las siguientes condiciones iniciales:

Figura 1

Envolvente de la vivienda

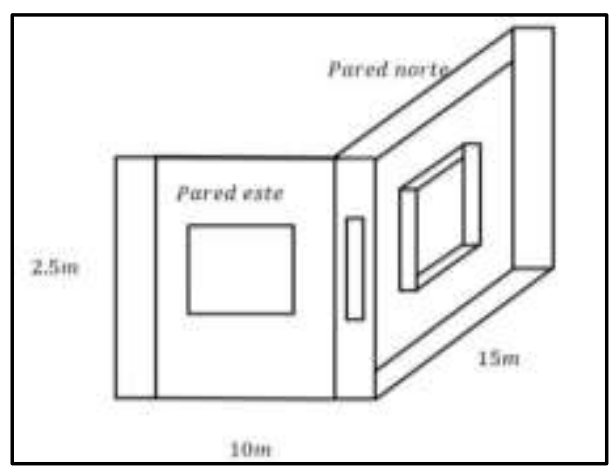

Fuente: Vivieda (2015)

Tabla 1

Condiciones iniciales de la vivienda

\begin{tabular}{lcc}
\hline NOMECLATURA OCTAVE & SIMBOLOGIA & VALOR \\
\hline \#Temperatura interior & T_inf_i & 23.00 \\
\#Temperatura exterior $26.2-30.5-32.4$ & T_inf_0 & 26.20 \\
\#Temperatura promedio anual del piso & T_inf_1 & 23.00 \\
\hline
\end{tabular}


Tabla 1

Condiciones iniciales de la vivienda (continuación)

\begin{tabular}{lcc}
\hline NOMECLATURA OCTAVE & SIMBOLOGIA & VALOR \\
\hline \#Espesor de bloque de concreto de pared & Lb & 0.15 \\
\#Espesor de vidrio de ventana & $\mathrm{Lv}$ & 0.006 \\
\#Espesor del piso & $\mathrm{Lp}$ & 0.20 \\
\#Espesor del pino & $\mathrm{Lpi}$ & 0.050 \\
\#Espesor del enlucido & $\mathrm{Le}$ & 0.010 \\
\#Conductividad de bloque de concreto de pared & $\mathrm{kb}$ & 0.49 \\
\#Conductividad de vidrio de ventana & $\mathrm{kv}$ & 1.05 \\
\#Conductividad de loza & $\mathrm{kl}$ & 0.23 \\
\#Conductividad del piso & $\mathrm{kp}$ & 1.40 \\
\#Conductividad del pino & $\mathrm{kpi}$ & 0.163 \\
\#Conductividad del enlucido & $\mathrm{ke}$ & 0.90 \\
\#Coeficiente de conveccion del aire interior & $\mathrm{h} \_\mathrm{i}$ & 4.00 \\
\#Coeficiente de conveccion del aire exterior & $\mathrm{h} \_\mathrm{o}$ & 28.373 \\
\#Área de pared norte - area de ventana & An & 25.00 \\
\#Área total de pared sur & As & 37.50 \\
\#Área de pared norte - area de ventana & Ae & 12.50 \\
\#Área total de pared oeste & Ao & 25.00 \\
\#Área de ventana Norte y este & Av & 12.50 \\
\#Área loza & Al & 150.00 \\
\#Área del piso & Ap & 150.00 \\
\hline
\end{tabular}

Fuente: Vivienta (2018)

\section{Pared plana}

La conducción de calor atreves de una pared plana se puede reacomodar para tener resistencias térmicas de la pared en contra de la conducción de calor o simplemente la resistencia a la conducción de la pared. Es importante comprender que la resistencia térmica de un medio depende de la configuración geométrica y de las propiedades térmicas del medio (Arenas, 2020). Como por lo general, en la mayoría de los casos los problemas de transmisión de calor, se reducen a la determinación de las cantidades de calor transferidas y temperaturas de los distintos planos.

\section{Resistencias térmicas}

La trasferencia de calor unidimensional en estado estacionario a través de una pared plana de espesor L, área A y conductividad térmica K que está expuesta ala convección sobre ambos lados hacia fluidos a las temperaturas T1 y T2, con coeficiente de trasferencia de calor h1 y h2, respectivamente, la variación de la temperatura varia en forma lineal en condiciones estacionarias (Sánchez, 2014). 
Figura 2

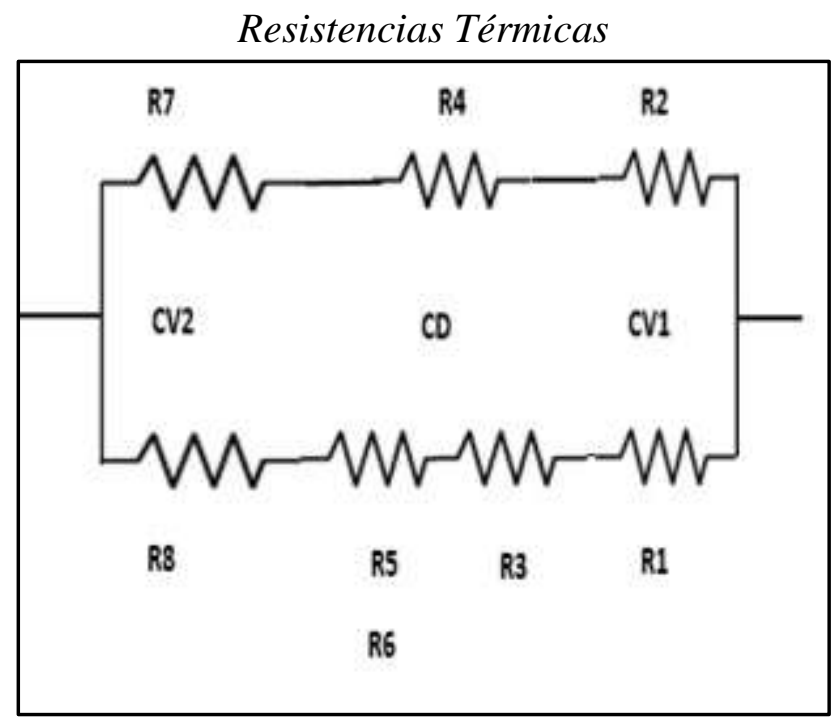

Fuente: Cengel (2007)

\section{Metodología}

El enfoque de la investigación es cuantitativo, mediante un proceso secuencial y probatorio. Cada etapa precede a la siguiente es decir definir la ganancia de calor mediante la definición de las variables como son las condiciones iniciales de la vivienda, para luego realizar la modelación atendiendo los criterios de resistencias térmicas en seri y paralelos analizando los parámetros de convección y conducción. El orden es riguroso, aunque podemos redefinir alguna fase.

El diseño de la investigación es de tipo exploratorio, esto se justifica ya que el objetivo es examinar un tema o problema de investigación poco estudiado como es las ganancias de calor de una vivienda de carácter social que implica un segmento de la población históricamente excluidos es decir, cuando la revisión de la literatura reveló que tan solo hay guías no investigadas como son los datos de ministerio urbano y vivienda, con este documento se motiva a indagar sobre el tema mediante nuevas perspectivas. Los estudios exploratorios sirven para familiarizarnos con fenómenos relativamente desconocidos, obtener información sobre la posibilidad de llevar a cabo una investigación más completa respecto de un contexto particular, indagar nuevos problemas, identificar conceptos o variables promisorias, establecer prioridades para investigaciones futuras, o sugerir afirmaciones y postulados (Sampieri, 2014).

Las características de la investigación definida en el presente documento, refiere la necesidad de medir y estimar magnitudes de la ganancia de calor para luego asociarla con los valores requeridos para que la vivienda resulte eco-eficiente. También se ha delimitados el estudio térmico de la vivienda de interés social en la ciudad de Guayaquil a conocer de forma concreta cómo se comporta a una temperatura promedio diaria es decir al medio día cuando el calor sensible es evidente por las características de la zona. Mediante la revisión de los datos establecidos por el instituto meteorológico y algunas 
características térmicas de los materiales comunes de construcción como el concreto liviano y bloque se derivó el estudio de forma exploratoria para examinar mediante la modelación en octave para obtener datos específicos que nos permiten conocer las ganancias de calor por conducción y convección.

La obtención de los datos se fundamenta, en la medición de la temperatura interna de la vivienda además de la temperatura en el exterior; el tamaño de la envolvente se caracterizó mediante la longitud de (ancho y largo). Los procedimientos son estandarizados ya que constan en tablas y las fórmulas están en el libro de Increpara.

Algunos informes de investigación se centran en la explicación de algún tema de interés. Este objetivo implica conclusiones indeterminadas, o bien, tentativas; por eso es importante que esto se indique. Los lectores deben saber que la intención es solamente indagar el estadio de una situación específica. Por lo general, este tipo de investigación es útil en la toma de decisiones para proponer la vivienda como ecoeficiente. Métodos de investigación, destinados a descubrir nuevas verdades, a esclarecer hechos desconocidos o a enriquecer el patrimonio de conocimientos, pueden ser de investigación religiosa, filosófica o científica, de acuerdo con el mundo de valores o hechos que se pretenda esclarecer (Guerrero, 2016).

\section{Resultados y discusión}

Cálculo de las ganancias de calor por paredes, suelo y techo, a las $09 \mathrm{~h} 00$, aplicación "ONE NOTE” de Microsoft.

Resistencias en serie de la pared este

$$
\begin{aligned}
\mathrm{R}_{\text {pared, conv1 }} & =\frac{1}{\mathrm{~h}_{0} * \mathrm{~A}_{\mathrm{E}}}=\frac{1}{28.373\left[\frac{\mathrm{W}}{\mathrm{m}^{2} \mathrm{~K}}\right] * 12.5\left[\mathrm{~m}^{2}\right]}=2.819 \times 10^{-3}\left[\frac{\mathrm{K}}{\mathrm{W}}\right] \\
\mathrm{R}_{\text {pared,cond1 }} & =\frac{\mathrm{L}_{\mathrm{B}}}{\mathrm{K}_{\mathrm{B}} * \mathrm{~A}_{\mathrm{E}}}=\frac{0.15[\mathrm{~m}]}{0.49\left[\frac{\mathrm{W}}{\mathrm{mK}}\right] * 12.5\left[\mathrm{~m}^{2}\right]}=0.024490\left[\frac{\mathrm{K}}{\mathrm{W}}\right] \\
\mathrm{R}_{\text {pared,conv3 }} & =\frac{1}{\mathrm{~h}_{1} * \mathrm{~A}_{\mathrm{E}}}=\frac{1}{4\left[\frac{\mathrm{w}}{\mathrm{m}^{2} \mathrm{~K}}\right] * 12.5\left[\mathrm{~m}^{2}\right]}=2 \times 10^{-2}\left[\frac{\mathrm{K}}{\mathrm{W}}\right]
\end{aligned}
$$

Resistencias en serie de la ventana

$$
\begin{aligned}
& \mathrm{R}_{\mathrm{vent}, \text { conv2 }}=\frac{1}{\mathrm{~h}_{0} * \mathrm{~A}_{\mathrm{v}}}=\frac{1}{28.373\left[\frac{\mathrm{w}}{\mathrm{m}^{2} \mathrm{~K}}\right] * 12.5\left[\mathrm{~m}^{2}\right]}=2.819 \times 10^{-3}\left[\frac{\mathrm{K}}{\mathrm{W}}\right] \\
& \mathrm{R}_{\mathrm{vent}, \text { cond } 2}=\frac{\mathrm{L}_{\mathrm{v}}}{\mathrm{K}_{\mathrm{v}} * \mathrm{~A}_{\mathrm{v}}}=\frac{0.006[\mathrm{~m}]}{1.05\left[\frac{\mathrm{W}}{\mathrm{mK}}\right] * 12.5\left[\mathrm{~m}^{2}\right]}=4.57 \times 10^{-4}\left[\frac{\mathrm{K}}{\mathrm{W}}\right] \\
& \mathrm{R}_{\mathrm{vent}, \text { conv } 4}=\frac{1}{\mathrm{~h}_{1} * \mathrm{~A}_{\mathrm{v}}}=\frac{1}{4\left[\frac{\mathrm{W}}{\mathrm{m}^{2} \mathrm{~K}}\right] * 12.5\left[\mathrm{~m}^{2}\right]}=2 \times 10^{-2}\left[\frac{\mathrm{K}}{\mathrm{W}}\right]
\end{aligned}
$$


Cálculo de las resistencias en paralelo de la pared con la ventana

Suma de Resistencias en serie de la pared

$$
\mathrm{R}_{\text {tot }, \mathrm{p}}=\left(2.819 \times 10^{-3}+0.024490+2 \times 10^{-2}\right)\left[\frac{\mathrm{K}}{\mathrm{W}}\right]=4.7309 \times 10^{-2}\left[\frac{\mathrm{K}}{\mathrm{W}}\right]
$$

Suma de Resistencias en serie de la ventana

$\mathrm{R}_{\text {tot }, \mathrm{v}}=\left(2.819 \times 10^{-3}+4.57 \times 10^{-4}+2 \times 10^{-2}\right)\left[\frac{\mathrm{K}}{\mathrm{W}}\right]=2.3277 \times 10^{-2}\left[\frac{\mathrm{K}}{\mathrm{W}}\right]$

Suma de resistencias en paralelo

$$
\begin{aligned}
\frac{1}{\mathrm{R}_{\mathrm{TOT}}} & =\frac{1}{\mathrm{R}_{\text {tot, } \mathrm{p}}}+\frac{1}{\mathrm{R}_{\mathrm{tot}, \mathrm{v}}}=>\mathrm{R}_{\mathrm{TOT}}=\left(\frac{1}{\mathrm{R}_{\mathrm{tot}, \mathrm{p}}}+\frac{1}{\mathrm{R}_{\mathrm{tot}, \mathrm{v}}}\right)^{\wedge}-1 \\
\mathrm{R}_{\mathrm{TOT}} & =\left(\frac{1}{4.7309 \times 10^{-2}}+\frac{1}{2.3277 \times 10^{-2}}\right)^{\wedge}-1>\mathrm{R}_{\mathrm{eq}, 1}=1.5601 \times 10^{-2}\left[\frac{\mathrm{K}}{\mathrm{W}}\right]
\end{aligned}
$$

Calculo del flujo de calor en la pared este

$$
\mathrm{q}_{\mathrm{E}}=\frac{\left(\mathrm{T}_{\infty 0}-\mathrm{T}_{\infty 1}\right)}{\mathrm{R}_{\mathrm{TOT}}} \Rightarrow \frac{(26.2-23)[\mathrm{K}]}{1.5601 \times 10^{-2}\left[\frac{\mathrm{K}}{\mathrm{W}}\right]}=205.12 \mathrm{~W}
$$

Cálculo de flujo de calor en la pared sur

Resistencias en serie de la pared sur

$$
\begin{aligned}
\mathrm{R}_{\text {pared,conv1 }} & =\frac{1}{\mathrm{~h}_{0} * \mathrm{~A}_{\mathrm{S}}}=\frac{1}{28.373\left[\frac{\mathrm{W}}{\mathrm{m}^{2} \mathrm{~K}}\right] * 37.5\left[\mathrm{~m}^{2}\right]}=9.3986 \times 10^{-4}\left[\frac{\mathrm{K}}{\mathrm{W}}\right] \\
\mathrm{R}_{\text {pared,cond1 }} & =\frac{\mathrm{L}_{\mathrm{B}}}{\mathrm{K}_{\mathrm{B}} * \mathrm{~A}_{\mathrm{S}}}=\frac{0.15[\mathrm{~m}]}{0.49\left[\frac{\mathrm{w}}{\mathrm{mK}}\right] * 37.5\left[\mathrm{~m}^{2}\right]}=8.1633 \times 10^{-3}\left[\frac{\mathrm{K}}{\mathrm{W}}\right] \\
\mathrm{R}_{\text {pared,conv2}} & =\frac{1}{\mathrm{~h}_{1} * \mathrm{~A}_{\mathrm{S}}}=\frac{1}{4\left[\frac{\mathrm{w}}{\mathrm{m}^{2} \mathrm{~K}}\right] * 37.5\left[\mathrm{~m}^{2}\right]}=6.6667 \times 10^{-3}\left[\frac{\mathrm{K}}{\mathrm{W}}\right]
\end{aligned}
$$

Suma total de las resistencias

$$
\begin{aligned}
& \mathrm{R}_{\mathrm{TOT}}=\mathrm{R}_{\text {pared, conv1 }}+\mathrm{R}_{\text {pared,cond1 }}+\mathrm{R}_{\text {pared, conv2 }} \\
& \mathrm{R}_{\mathrm{TOT}}=\left(9.3986 \times 10^{-4}+8.1633 \times 10^{-3}+6.6667 \times 10^{-3}\right)\left[\frac{\mathrm{K}}{\mathrm{W}}\right]=1.5770 \times 10^{-2}\left[\frac{\mathrm{K}}{\mathrm{W}}\right]
\end{aligned}
$$

Calculo del flujo de calor en la pared sur

$$
\mathrm{q}_{\mathrm{S}}=\frac{\left(\mathrm{T}_{\infty 0}-\mathrm{T}_{\infty 1}\right)}{\mathrm{R}_{\mathrm{TOT}}} \Rightarrow \frac{(26.2-23)[\mathrm{K}]}{1.5770 \times 10^{-2}\left[\frac{\mathrm{K}}{\mathrm{W}}\right]}=202.92 \mathrm{~W}
$$

Cálculo de flujo de calor en la pared oeste

Resistencias en serie de la pared oeste 
$\mathrm{R}_{\text {pared, conv1 }}=\frac{1}{\mathrm{~h}_{0} * \mathrm{~A}_{\mathrm{E}}}=\frac{1}{28.373\left[\frac{\mathrm{W}}{\mathrm{m}^{2} \mathrm{~K}}\right] * 25\left[\mathrm{~m}^{2}\right]}=1.4098 \times 10^{-3}\left[\frac{\mathrm{K}}{\mathrm{W}}\right]$

El resultado obtenido a estas condiciones es de 1409x10-3 K/W.

Así mismo, para el cálculo de pared oeste por conducción detallamos:

$\mathrm{R}_{\text {pared,cond1 }}=\frac{\mathrm{L}_{\mathrm{B}}}{\mathrm{K}_{\mathrm{B}} * \mathrm{~A}_{\mathrm{E}}}=\frac{0.15[\mathrm{~m}]}{0.49\left[\frac{\mathrm{W}}{\mathrm{mK}}\right] * 25\left[\mathrm{~m}^{2}\right]}=0.012245\left[\frac{\mathrm{K}}{\mathrm{W}}\right]$

Obtenemos el resultado de $0.012245 \mathrm{~K} / \mathrm{W}$, y finalmente, para el cálculo de resistencias en serie de la pared oeste, por convección expresamos:

$\mathrm{R}_{\text {pared,conv2 }}=\frac{1}{\mathrm{~h}_{1} * \mathrm{~A}_{\mathrm{E}}}=\frac{1}{4\left[\frac{\mathrm{W}}{\mathrm{m}^{2} \mathrm{~K}}\right] * 25\left[\mathrm{~m}^{2}\right]}=0.010000\left[\frac{\mathrm{K}}{\mathrm{W}}\right]$

Conseguimos el resultado de $0.010000 \mathrm{~K} / \mathrm{W}$ : Por lo tanto, la sumatoria total de las resistencias en la pared oeste siendo las 09h00 obtenemos con la siguiente expresión

Suma total de las resistencias

$$
\begin{aligned}
& \mathrm{R}_{\mathrm{TOT}}=\mathrm{R}_{\text {pared, conv1 }}+\mathrm{R}_{\text {pared,cond1 }}+\mathrm{R}_{\text {pared, conv2 }} \\
& \mathrm{R}_{\mathrm{TOT}}=\left(1.4098 \times 10^{-3}+0.012245+0.010000\right)\left[\frac{\mathrm{K}}{\mathrm{W}}\right]=2.3655 \times 10^{-2}\left[\frac{\mathrm{K}}{\mathrm{W}}\right]
\end{aligned}
$$

La sumatoria de las resistencias por conversión y conducción en la pared oeste siendo las $09 \mathrm{~h} 00$ es de $2.3655 \times 10-2 \mathrm{~K} / \mathrm{W}$, ahora.

Para el cálculo del flujo de calor en la pared oeste, se detalla a continuación.

Calculo del flujo de calor en la pared oeste

$$
\mathrm{q}_{\mathrm{o}}=\frac{\left(\mathrm{T}_{\infty 0}-\mathrm{T}_{\infty 1}\right)}{\mathrm{R}_{\mathrm{TOT}}} \Rightarrow \frac{(26.2-23)[\mathrm{K}]}{2.3655 \times 10^{-2}\left[\frac{\mathrm{K}}{\mathrm{W}}\right]}=135.28 \mathrm{~W}
$$

Por lo tanto, el flujo de calor en la pared oeste siendo las $09 \mathrm{~h} 00$ es de $135.28 \mathrm{~W}$.

Cálculo de flujo de calor en la loza

Resistencias en serie de la loza

$\mathrm{R}_{\text {conv1 }}=\frac{1}{\mathrm{~h}_{0} * \mathrm{~A}_{\mathrm{L}}}=\frac{1}{28.373\left[\frac{\mathrm{W}}{\mathrm{m}^{2} \mathrm{~K}}\right] * 150\left[\mathrm{~m}^{2}\right]}=2.3497 \times 10^{-4}\left[\frac{\mathrm{K}}{\mathrm{W}}\right]$

Ahora, el siguiente calculo para determinar la resistencia de la loza por conducción expresamos lo siguiente: 
$\mathrm{R}_{\text {cond } 1}=\frac{\mathrm{L}_{\mathrm{B}}}{\mathrm{K}_{\mathrm{B}} * \mathrm{~A}_{\mathrm{L}}}=\frac{0.15[\mathrm{~m}]}{0.23\left[\frac{\mathrm{W}}{\mathrm{mK}}\right] * 150\left[\mathrm{~m}^{2}\right]}=4.3478 \times 10^{-4}\left[\frac{\mathrm{K}}{\mathrm{W}}\right]$

El resultado logrado en la loza por conducción es de 4.3478x 『10》^(-4) K/W, y.

Finalmente, para el cálculo de la resistencia por convección expresamos lo siguiente:

$\mathrm{R}_{\mathrm{conv2}}=\frac{1}{\mathrm{~h}_{1} * \mathrm{~A}_{\mathrm{L}}}=\frac{1}{4\left[\frac{\mathrm{w}}{\mathrm{m}^{2} \mathrm{~K}}\right] * 150\left[\mathrm{~m}^{2}\right]}=1.6667 \times 10^{-3}\left[\frac{\mathrm{K}}{\mathrm{W}}\right]$

El resultado conseguido por convección en la loza siendo las $09 \mathrm{~h} 00$ es de $1.6667 \mathrm{x}$ 『10 $\rrbracket \wedge(-3) \mathrm{K} / \mathrm{W}$.

Ahora, para el cálculo de sumatoria total de resistencias en la loza se detalla lo siguiente:

Suma total de las resistencias

$$
\begin{aligned}
& \mathrm{R}_{\mathrm{TOT}}=\mathrm{R}_{\text {conv } 1}+\mathrm{R}_{\text {cond } 1}+\mathrm{R}_{\text {conv2 }} \\
& \mathrm{R}_{\mathrm{TOT}}=\left(2.3497 \times 10^{-4}+4.3478 \times 10^{-4}+1.6667 \times 10^{-3}\right)\left[\frac{\mathrm{K}}{\mathrm{W}}\right]=6.2495 \times 10^{-3}\left[\frac{\mathrm{K}}{\mathrm{W}}\right]
\end{aligned}
$$

Por lo tanto, la sumatoria total de las resistencias en la loza a las $09 \mathrm{~h} 00$ es de $6.2495 \mathrm{x}$ 『 10】^(-3) K/W.

Para el cálculo del flujo de calor en la loza, se detalla a continuación.

\section{Calculo del flujo de calor en la loza}

$$
\mathrm{q}_{\mathrm{L}}=\frac{\left(\mathrm{T}_{\infty 0}-\mathrm{T}_{\infty 1}\right)}{\mathrm{R}_{\mathrm{TOT}}} \Rightarrow \frac{(26.2-23)[\mathrm{K}]}{6.2495 \times 10^{-3}\left[\frac{\mathrm{K}}{\mathrm{W}}\right]}=512.04 \mathrm{~W}
$$

Por lo tanto, el flujo de calor en la loza siendo las $09 \mathrm{~h} 00$ es de $512.04 \mathrm{~W}$.

Cálculo de flujo de calor en el piso

Resistencias en serie del piso

$$
\mathrm{R}_{\text {conv1 }}=\frac{1}{\mathrm{~h}_{0} * \mathrm{~A}_{\mathrm{L}}}=\frac{1}{4\left[\frac{\mathrm{W}}{\mathrm{m}^{2} \mathrm{~K}}\right] * 150\left[\mathrm{~m}^{2}\right]}=1.6667 \times 10^{-3}\left[\frac{\mathrm{K}}{\mathrm{W}}\right]
$$

Para este caso, cálculo de resistencias en serie en el piso de la vivienda, por convección siendo las 09h00 tenemos un valor de $1.6667 \times 10-3 \mathrm{~K} / \mathrm{W}$ :

$$
\mathrm{R}_{\text {cond } 1}=\frac{\mathrm{L}_{\mathrm{B}}}{\mathrm{K}_{\mathrm{p}} * \mathrm{~A}_{\mathrm{L}}}=\frac{0.20[\mathrm{~m}]}{1.40\left[\frac{\mathrm{W}}{\mathrm{mK}}\right] * 150\left[\mathrm{~m}^{2}\right]}=9.5238 \times 10^{-4}\left[\frac{\mathrm{K}}{\mathrm{W}}\right]
$$

Así mismo, en la misma hora para el cálculo de resistencias en serie por conducción en el piso de la vivienda, tenemos un valor de $1.6667 \times 10-3 \mathrm{~K} / \mathrm{W}$ : 
Ahora la sumatoria total de las resistencias en el piso de la vivienda expresamos lo siguiente:

Suma total de las resistencias

$$
\begin{aligned}
& \mathrm{R}_{\mathrm{TOT}}=\mathrm{R}_{\text {conv } 1}+\mathrm{R}_{\text {cond } 1} \\
& \mathrm{R}_{\mathrm{TOT}}=\left(1.6667 \times 10^{-3}+9.5238 \times 10^{-4}\right)\left[\frac{\mathrm{K}}{\mathrm{W}}\right]=2.6190 \times 10^{-3}\left[\frac{\mathrm{K}}{\mathrm{W}}\right]
\end{aligned}
$$

Por lo tanto, el resultado total de sumatoria de resistencias en el piso de la vivienda a las $09 \mathrm{~h} 00$ es de $2.6190 \mathrm{x} \llbracket 10 \rrbracket \wedge(-3) \mathrm{K} / \mathrm{W}$.

Para el cálculo del flujo de calor en el piso de la vivienda a las 09h00, se detalla a continuación.

Cálculo del flujo de calor en el piso

$\mathrm{q}_{\mathrm{p}}=\frac{\left(\mathrm{T}_{\infty 0}-\mathrm{T}_{\infty 1}\right)}{\mathrm{R}_{\mathrm{TOT}}} \Rightarrow \frac{(26.2-23)[\mathrm{K}]}{2.6190 \times 10^{-3}\left[\frac{\mathrm{K}}{\mathrm{W}}\right]}=1336.4 \mathrm{~W}$

Por lo tanto, el flujo de calor en el piso siendo las $09 \mathrm{~h} 00$ es de $1336.4 \mathrm{~W}$.

Ganancia total de calor a las $09 \mathrm{~h} 00,12 \mathrm{~h} 00$ y $15 \mathrm{~h} 00$, sin aislamiento y con 2 propuestas, aplicación "ONE NOTE” de Microsoft.

Cálculo del flujo de calor en la vivienda a las 9am

$\mathrm{q}_{\mathrm{TOT}}=\mathrm{q}_{\mathrm{N}}+\mathrm{q}_{\mathrm{E}}+\mathrm{q}_{\mathrm{S}}+\mathrm{q}_{\mathrm{O}}+\mathrm{q}_{\mathrm{L}}+\mathrm{q}_{\mathrm{P}}$

$\mathrm{q}_{\text {тот }}=2664.52 \mathrm{~W}$

La sumatoria de las ganancias de calor de cada uno de los lados de la vivienda (paredes, loza y piso) a las 9:00 horas es de 2664.52 Wattios.

Cálculo del flujo de calor en la vivienda a las $12 \mathrm{pm}$

$\mathrm{q}_{\mathrm{TOT}}=\mathrm{q}_{\mathrm{N}}+\mathrm{q}_{\mathrm{E}}+\mathrm{q}_{\mathrm{S}}+\mathrm{q}_{\mathrm{O}}+\mathrm{q}_{\mathrm{L}}+\mathrm{q}_{\mathrm{P}}$

$\mathrm{q}_{\text {тот }}=4449.1 \mathrm{~W}$

La sumatoria de las ganancias de calor de cada uno de los lados de la vivienda (paredes, loza y piso) a las 12:00 horas es de 4449.1 Wattios.

Cálculo del flujo de calor en la vivienda a las $15 \mathrm{pm}$

$\mathrm{q}_{\mathrm{TOT}}=\mathrm{q}_{\mathrm{N}}+\mathrm{q}_{\mathrm{E}}+\mathrm{q}_{\mathrm{S}}+\mathrm{q}_{\mathrm{O}}+\mathrm{q}_{\mathrm{L}}+\mathrm{q}_{\mathrm{P}}$

$\mathrm{q}_{\text {тот }}=5237.7 \mathrm{~W}$

La sumatoria de las ganancias de calor de cada uno de los lados de la vivienda (paredes, loza y piso) a las 15:00 horas, es de 5237.7 Wattios 
Para presentar las viviendas como ecoeficientes, se deben presentar las propuestas en las cuales las ganancias de calor incidentes en las viviendas a las horas ya establecidas sean mínimas.

\section{Primera propuesta}

Paredes enlucidas con mortero (arena y cemento) el cual tiene un coeficiente de conductividad de $0,90 \mathrm{~W} / \mathrm{mK}$

Cálculo del flujo de calor en la vivienda a las 9 am con enlucido

$\mathrm{q}_{\mathrm{TOT}}=\mathrm{q}_{\mathrm{N}}+\mathrm{q}_{\mathrm{E}}+\mathrm{q}_{\mathrm{S}}+\mathrm{q}_{\mathrm{O}}+\mathrm{q}_{\mathrm{L}}+\mathrm{q}_{\mathrm{P}}$

$\mathrm{q}_{\mathrm{TOT}}=2611.7 \mathrm{~W}$

Se reduce solo $2 \%$ de flujo de calor

La sumatoria de las ganancias de calor de cada uno de los lados de la vivienda (paredes, loza y piso) a las 9:00 horas, con paredes interiores enlucidas es de 2611.7 Wattios, con lo cual se logra una reducción del $2 \%$ de ganancia de calor en la vivienda con respecto a la ganancia de calor a la misma hora sin el enlucido.

Cálculo del flujo de calor en la vivienda a las $12 \mathrm{pm}$ con enlucido

$\mathrm{q}_{\mathrm{TOT}}=\mathrm{q}_{\mathrm{N}}+\mathrm{q}_{\mathrm{E}}+\mathrm{q}_{\mathrm{S}}+\mathrm{q}_{\mathrm{O}}+\mathrm{q}_{\mathrm{L}}+\mathrm{q}_{\mathrm{P}}$

$\mathrm{q}_{\mathrm{TOT}}=4374.9 \mathrm{~W}$

Se reduce solo $2 \%$ de flujo de calor

La sumatoria de las ganancias de calor de cada uno de los lados de vivienda (paredes, loza y piso) a las 12:00 horas, con paredes interiores enlucidas es de 4374.9 Wattios, con lo cual se logra una reducción del $2 \%$ de ganancia de calor en la vivienda con respecto a la ganancia de calor a la misma hora sin el enlucido.

Cálculo del flujo de calor en la vivienda a las $15 \mathrm{pm}$ con enlucido

$\mathrm{q}_{\mathrm{TOT}}=\mathrm{q}_{\mathrm{N}}+\mathrm{q}_{\mathrm{E}}+\mathrm{q}_{\mathrm{S}}+\mathrm{q}_{\mathrm{O}}+\mathrm{q}_{\mathrm{L}}+\mathrm{q}_{\mathrm{P}}$

$\mathrm{q}_{\mathrm{TOT}}=5154.0 \mathrm{~W}$

Se reduce solo $2 \%$ de flujo de calor

La sumatoria de las ganancias de calor de cada uno de los lados de vivienda (paredes, loza y piso) a las 15:00 horas, con paredes interiores enlucidas es de 5153.0 Wattios, con lo cual se logra una reducción del $2 \%$ de ganancia de calor en la vivienda con respecto a la ganancia de calor a la misma hora sin el enlucido.

\section{Segunda propuesta}

Paredes revestidas con madera (pino) el cual tiene un coeficiente de conductividad de $0,163 \mathrm{~W} / \mathrm{mK}$

Cálculo del flujo de calor en la vivienda a las 9am con madera de pino en las paredes 


$$
\mathrm{q}_{\mathrm{TOT}}=\mathrm{q}_{\mathrm{N}}+\mathrm{q}_{\mathrm{E}}+\mathrm{q}_{\mathrm{S}}+\mathrm{q}_{\mathrm{O}}+\mathrm{q}_{\mathrm{L}}+\mathrm{q}_{\mathrm{P}}
$$

$\mathrm{q}_{\mathrm{TOT}}=1767.5 \mathrm{~W}$

Se reduce solo $33.66 \%$ de flujo de calor

La sumatoria de las ganancias de calor de cada uno de los lados de la vivienda (paredes, loza y piso) a las 9:00 horas, con paredes exteriores revestidas con madera es de 1767.5 Wattios, con lo cual se logra una reducción del $33.66 \%$ de ganancia de calor en la vivienda con respecto a la ganancia de calor a la misma hora sin el enlucido y el revestimiento.

Calculo del flujo de calor en la vivienda a las $12 \mathrm{pm}$ con madera de pino en las paredes

$\mathrm{q}_{\mathrm{TOT}}=\mathrm{q}_{\mathrm{N}}+\mathrm{q}_{\mathrm{E}}+\mathrm{q}_{\mathrm{S}}+\mathrm{q}_{\mathrm{O}}+\mathrm{q}_{\mathrm{L}}+\mathrm{q}_{\mathrm{P}}$

$\mathrm{q}_{\text {TOT }}=3134.1 \mathrm{~W}$

Se reduce solo $29.55 \%$ de flujo de calor

La sumatoria de las ganancias de calor de cada uno de los lados de la vivienda (paredes, loza y piso) a las 12:00 horas, con paredes exteriores revestidas con madera es de 3134.1 Wattios, con lo cual se logra una reducción del $29.55 \%$ de ganancia de calor en la vivienda con respecto a la ganancia de calor a la misma hora sin el enlucido y el revestimiento.

Cálculo del flujo de calor en la vivienda a las 15pm con madera de pino en las paredes

$$
\mathrm{q}_{\mathrm{TOT}}=\mathrm{q}_{\mathrm{N}}+\mathrm{q}_{\mathrm{E}}+\mathrm{q}_{\mathrm{S}}+\mathrm{q}_{\mathrm{O}}+\mathrm{q}_{\mathrm{L}}+\mathrm{q}_{\mathrm{P}}
$$

$\mathrm{q}_{\mathrm{TOT}}=3738.0 \mathrm{~W}$

Se reduce solo $28.63 \%$ de flujo de calor

La sumatoria de las ganancias de calor de cada uno de los lados de la vivienda (paredes, loza y piso) a las 15:00 horas, con paredes exteriores revestidas con madera es de 3738.0 Wattios, con lo cual se logra una reducción del $28.63 \%$ de ganancia de calor en la vivienda con respecto a la ganancia de calor a la misma hora sin el enlucido y el revestimiento.

El resumen de las actividades de cálculo realizados, se detalla en la tabla 2.

Tabla 2

Ganancias de calor con las diferentes propuestas

\begin{tabular}{cccccccccc}
\hline CONDICIONES & HORA & $\mathbf{Q}$ & $\%$ & HORA & $\mathbf{Q}$ & $\%$ & HORA & Q & $\%$ \\
\hline NORMAL & & 2664.5 & - & & 4449.1 & - & & 5237.7 & - \\
PROPUESTA 1 & $09 \mathrm{~h} 00$ & 2611.7 & 2 & $12 \mathrm{~h} 00$ & 4374.9 & 2 & $15 \mathrm{~h} 00$ & 5154.0 & 2 \\
PROPUESTA 2 & & 1767.5 & 33.66 & & 3134.1 & 29.55 & & 738.0 & 28.6 \\
\hline
\end{tabular}


Por lo tanto, en referencia, $a$.

- La propuesta 1, consta de un mortero para enlucido de paredes, preparado con arena y cemento. Con un espesor de $10 \mathrm{~mm}$ con un coeficiente de conductividad $\mathrm{k}$ igual a $0,90 \mathrm{~W} / \mathrm{mK}$. A ser aplicado como enlucido en las paredes internas de la vivienda, donde se logra alcanzar una reducción del $2 \%$ de ganancia de calor en a vivienda.

- La propuesta 2, consta de pablas de pino, con un espesor de $50 \mathrm{~mm}$, con un coeficiente de conductividad $\mathrm{k}$ igual a $0,163 \mathrm{~W} / \mathrm{mK}$. A ser aplicado como revestimiento sobre el bloque en las paredes externas de la vivienda, donde se logra alcanzar una reducción del promedio de $30 \%$ de ganancia de calor en a vivienda.

\section{Conclusiones}

Se determinó que la posición del sol con respecto a la pared de la casa es un determinante para la ganancia de calor. Es importante que el constructor tome en cuenta esta variable para permitir exponer las áreas de la vivienda que tengan menor cantidad de metros cuadrados a la radiación del sol.

- Considerando que las condiciones climáticas en la ciudad de guayaquil tienen como principales características altos niveles de insolación, sería importante el uso de materiales para recubrimiento como pintura altamente reflectiva y emisivos, esto puede ser una ayuda sencilla y económica para reducir fuertemente la incidencia de la radiación solar en las superficies de la vivienda reflejándose en la reducción del consumo de energía para climatización de la casa.

- Se puede colocar una envolvente al exterior de la vivienda de un material que presente baja conductividad térmica como materiales aislantes que eviten la penetración al interior de radiación incidente del sol.

- Dependiendo de los espacios alrededor de las viviendas, jardineras, patios o áreas verdes colindantes a la misma, se podrían plantar árboles de manera distribuida, que permitan crear sombra y reducir de manera significativa la radiación solar en la vivienda. A más de ofrecer casa ecoeficientes también se puede presentar como eco ambientales.

La mejor propuesta para comercialización de las viviendas tipo eco-eficientes, ubicadas en la ciudad de Guayaquil, es el revestimiento de madera tipo pino, material que será utilizado en el interior de la vivienda para obtener una gran reducción calor de $33.66 \%$ a las $09 \mathrm{~h} 00,29.55 \%$ a las $12 \mathrm{~h} 00$ y finalmente a las $15 \mathrm{~h} 00$ una reducción de $28.63 \%$.

\section{Referencias bibliográficas}

Alvear, C, G. \&. (2021). Aplicación de la Ecuación d Balance Térmico para determinar el confort higrotérmico en la vivien unifamiliar en el cantón Girón. Ciencia Digital, 3. 

noviembre, 2021

Arenas, F. c. (2020). Transferencia de Calor. . Cordova: Impreso en Argentina - Printed in Argentina.

Calle, A. T. (3 de Marzo de 2021). Estrategias para diseño de vivienda social en Cuenca - Ecuador, mediante un análisis cuantitativo de patrones sociales, económicos, habitacionales y constructivos. Ciencia Digital., 4. doi:https://doi.org/10.33262/cienciadigital.v5i2.1595

Calle. C. C. (2021). Evaluación de estrategias bioclimáticas pasiv s para una vivienda de interés social ubicada en el cantón Morona en prospectiv a 50 años. Ciencia Digital , 2.

Cengel, Y. A. (2007). Transferencia de calo y masa. Mexico: MCgRAW-HILL.

Estevez, M. G. (2021). Analis comaprativo de aspectos termicos y resistencias mecánicas de los materiales y los elementos de la costrucción con tierra. Tecnologias medio anbientales y sostenibles., 140.

Filippín, M. \&. (2010). Comportamiento termico-energético. Obtenido de https://www.scielo.br/j/ac/a/dqShSBRW5YHSGfZCDknJrcC/?lang=es\&format $=\mathrm{pdf}$

Guerrero, L. (2016). Metodología de la. Londres: Universidad de Londres.

INCROPERA. (2011). Fundamentals os Heat and Mas Tansfer, Printed in the United States of. EEUU.

Medina. J. A. (2016), repositorio.ug.edu.e. Obtenido de repositorio.ug.edu.e: http://repositorio.ug.edu.ec/bitstream/redug/31563/2/TESIS.pdf

Olmedo, V. E. (Jujio de 2017). http://repositorio.puce.edu.ec/. Obtenido de http://repositorio.puce.edu.ec/bitstream/handle/22000/13770/tesis\%20completa. pdf? sequence $=1 \&$ isAllowed $=y$

Sampieri, R. H. (2014). Metodología de la Investigación. México D.F. doi:ISBN: 978$1-4562-2396-0$

Sánchez, J. A. (2014). Fundamentos de Transmisión de Calor. . España: Impreso en España. Printed in Spain.

Vivieda, M. d. (2015). Gia practica de la construcción para no profecionales. doi:ISBN.9942-951-51

Vivienta, M. d. (2018). Norma Ecuatoriana de la Construción NEC. Quito.

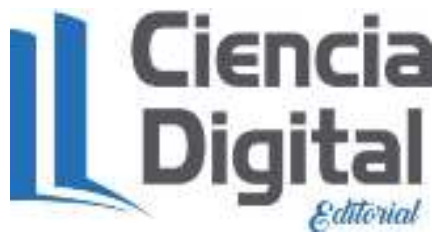




\section{PARA CITAR EL ARTÍCULO INDEXADO}

Intriago Mairongo, H. A., Gonzales Vega, A. A., Rosero Obando, E. M., \& Plaza Hernández, F. N. (2021). Estudio térmico en una vivienda situada en la ciudad de Guayaquil. AlfaPublicaciones, 3(4.1), 53-69. https://doi.org/10.33262/ap.v3i4.1.124

\section{Ciencia}

El artículo que se publica es de exclusiva responsabilidad de los autores y no necesariamente reflejan el pensamiento de la Revista Alfa Publicaciones.

El artículo queda en propiedad de la revista y, por tanto, su publicación parcial y/o total en otro medio tiene que ser autorizado por el director de la Revista Alfa Publicaciones.
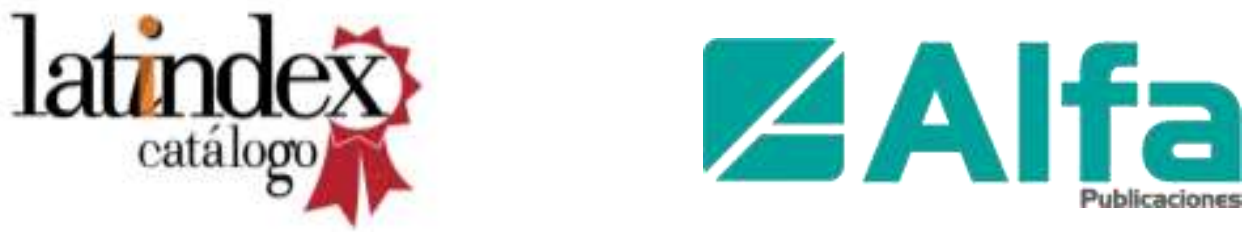